BMJ Open

Diabetes

Research

\& Care

\title{
Role of resistin, IL-6 and NH2-terminal portion proBNP in the pathogenesis of cardiac disease in type 2 diabetes mellitus
}

Samar Ebrahim Ghanem, ${ }^{1}$ Mohamed Abdel-Samiee $(1),{ }^{2}$ Mohamed Hamdy Torky, ${ }^{3}$ Ahmed Gaafar, ${ }^{4}$ Somia Mokabel Mohamed, ${ }^{5}$ Ghada Mohamed Mohamed Salah Eldin, ${ }^{5}$ Samah Mohammed Awad, ${ }^{6}$ Karema A Diab, ${ }^{7}$ Dalia M ELsabaawy, ${ }^{8}$ Sania Ali Yehia, ${ }^{9}$ Hany Abdelbary Abdelaziz Elbasyouni, ${ }^{10}$ Amr Aly Elshormilisy ${ }^{11}$

To cite: Ghanem SE, Abdel-Samiee M, Torky MH, et al. Role of resistin, IL-6 and $\mathrm{NH} 2$-terminal portion proBNP in the pathogenesis of cardiac disease in type 2 diabetes mellitus. BMJ Open Diab Res Care 2020;8:e001206. doi:10.1136/ bmjdrc-2020-001206

Received 17 January 2020 Revised 29 May 2020 Accepted 4 August 2020
Check for updates

\section{(C) Author(s) (or their} employer(s)) 2020. Re-use permitted under CC BY-NC. No commercial re-use. See rights and permissions. Published by BMJ.

For numbered affiliations see end of article.

Correspondence to Dr Mohamed Abdel-Samiee; drmohammed100@yahoo.com

\section{ABSTRACT}

Introduction Epidemiological and genetic studies have recorded the association between proinflammatory cytokines and the development of insulin resistance, diabetes, and cardiovascular disease. The role of interleukin 6 (IL-6), NH2terminal portion pro-brain natriuretic peptide (NT-proBNP) and resistin in the pathogenesis of heart disease in type 2 diabetes mellitus (T2DM) is still a matter of controversy. The current study aimed to evaluate the role of these biomarkers in the development of left ventricular systolic dysfunction and the ability to use them as non-invasive test in the prediction of left ventricular hypertrophy and systolic dysfunction in T2DM. Research design and methods 150 participants were included in this case-control study. Patients were divided into two subgroups according to echocardiographic findings: group 1a included 46 patients with type 2 diabetes mellitus and echocardiographic evidence of abnormal systolic function; group $1 \mathrm{~b}$ included 54 patients with type 2 diabetes mellitus and with normal echocardiogenic study; and group 2 included 50 apparently healthy controls. Routine laboratory investigations such as complete blood count, liver and renal function tests, and lipid profile, serum IL-6, NT-proBNP, and resistin were measured in all participants. Conventional echocardiography was done with special concern on the assessment of left ventricular systolic function (ejection fraction).

Results There was a significant increase in the level of resistin, NT-proBNP and IL-6 in group 1a patients compared with group $1 \mathrm{~b}$ and in healthy controls. Echocardiographic parameters showed a significant increase in left ventricular mass index, left ventricle posterior wall thickness, interventricular septum thickness, and left ventricle mass in group 1a compared with group $1 \mathrm{~b}$ and the control group. The increased left ventricular mass index was associated with higher levels of IL-6, NT-proBNP and resistin.

Conclusions Proinflammatory cytokines had a clear relation with left ventricular systolic dysfunction and hypertrophy and can be used as early non-invasive markers for detection of left ventricular remodeling and systolic dysfunction in patients with T2DM.

\section{INTRODUCTION}

The American Heart Association designated diabetes mellitus (DM) as a major risk factor for cardiovascular disease (CVD). CVD,

\section{Significance of this study}

What is already known about this subject?

- Epidemiological and genetic studies have recorded the association between proinflammatory cytokines and the development of insulin resistance, diabetes, and cardiovascular disease.

- The role of interleukin 6 (LL-6), NH2-terminal portion pro-brain natriuretic peptide (NT-proBNP) and resistin in the pathogenesis of heart disease in patients with type 2 diabetes mellitus is still a matter of controversy.

What are the new findings?

- There was a significant increase in the level of resistin, NT-proBNP and IL-6 in group 1a patients compared with group $1 \mathrm{~b}$ and in healthy controls.

- Echocardiographic parameters showed a significant increase in left ventricular mass index, left ventricle posterior wall thickness, interventricular septum thickness, and left ventricle mass in group 1a compared with group $1 \mathrm{~b}$ and the control group.

How might these results change the focus of research or clinical practice?

- Proinflammatory cytokines had a clear relation with left ventricular systolic dysfunction and hypertrophy and can be used as early non-invasive markers for detection of left ventricular remodeling and systolic dysfunction in patients with type 2 diabetes mellitus.

- Resistin, NT-proBNP, IL-6 and echocardiographic data and suggesting them as diagnostic markers are a subject of great biomedical importance and contribute to knowledge.

stroke, and peripheral vascular disease are the main macrovascular complications of $\mathrm{DM}^{1}$ Echocardiographic studies on large databases of patients with diabetic heart failure have shown an association with left ventricular hypertrophy (LVH), increased heart mass, and diastolic function. ${ }^{2}$ The effects of type 2 diabetes mellitus (T2DM) on the heart 
can be broadly grouped into two main categories: coronary heart disease (CHD) and diabetic cardiomyopathy (DCM), which is characterized by $\mathrm{LVH}$, left ventricular (LV) diastolic dysfunction, LV systolic dysfunction and cardiac autonomic neuropathy. ${ }^{3} 4$ The common final pathway for both CHD and DCM is clinical heart failure. ${ }^{5}$

Brain natriuretic peptide (BNP) is a potent vasodilator and a natriuretic factor regulating salt and water hemostasis. It is stored in the human cardiac tissue mainly as BNP-32, with a lesser amount of the precursor preproBNP. The circulating plasma forms of BNP are BNP-32 and the NH2-terminal portion proBNP (NT-proBNP). ${ }^{6}$ BNP and NT-proBNP may be used as a screening test for exercise-induced myocardial ischemia. ${ }^{7}$ In addition, plasma BNP in patients with systolic heart failure reflects the severity of diastolic abnormality. ${ }^{8}$ The diagnosis of heart failure due to diastolic dysfunction is usually based on typical symptoms and signs of heart failure in patients with preserved LV systolic function. ${ }^{9}$

As vascular inflammation has emerged as an important component of CVD, it is critical to elucidate the role of interleukin 6 (IL-6) in hypertension, endothelial dysfunction, ${ }^{10} \mathrm{CHD}$ and congestive heart failure with insulin resistance in T2DM. ${ }^{11}$ IL-6 level increases in CVD, including atherosclerosis, diabetes, heart failure, and hypertension. Its level is correlated positively with CVD progression. ${ }^{12}$

Resistin is a $12.5 \mathrm{kD}$ protein rich in cysteine and is produced mainly from activated white blood cells. ${ }^{13}$ It is a proinflammatory marker that is associated with insulin resistance, atherosclerosis and cardiac outcomes of DM. ${ }^{14}$ Resistin is produced and released from adipose tissue to serve endocrine functions likely involved in insulin resistance. This idea primarily stems from studies demonstrating that serum resistin levels increase with obesity and involved in energy metabolism and T2DM. Serum resistin levels have been found to decline with decreased adiposity following medical treatment. ${ }^{15}$

The purpose of our study was to identify the role of these biomarkers in the development of LV systolic dysfunction and the ability to use them as a non-invasive test in the prediction of $\mathrm{LVH}$ and systolic dysfunction in T2DM.

\section{RESEARCH DESIGN AND METHODS}

One hundred patients known to have T2DM were involved in this case-control study from January 2018 to March 2019, attending the cardiology outpatient clinic at Shebin El Kom Teaching Hospital and the outpatient clinic of the clinical hepatology and gastroenterology department at the National Liver Institute Hospital, Menoufia University. Patients were divided into two subgroups according to echocardiographic findings: group 1a included 46 patients with T2DM with left ventricular ejection fraction (LVEF $<50 \%$ ), and group $1 \mathrm{~b}$ included 54 patients with T2DM with normal echocardiographic study (LVEF $\geq 50 \%$ ). Fifty apparently healthy volunteers with age and sex matched to patients were enrolled in the study as a control group (group 2). The source of the controls was dependent on the source of the cases, such as their family relatives. Through systematic random sampling, one out of five individuals from the family relatives of the patients in group 1 was selected as the control.

DM was diagnosed according to WHO and the American Diabetes Association as fasting blood glucose $\geq 126 \mathrm{mg}$ / $\mathrm{dL}$ or 2-hour postprandial glucose $(\mathrm{PG}) \geq 200 \mathrm{mg} / \mathrm{dL} .^{16}$

Inclusion criteria included adults aged more than 30 years old and history of T2DM and on regular treatment.

Exclusion criteria included pregnancy, acute coronary syndrome, constrictive pericarditis, acute pericarditis, hypertrophic cardiomyopathy, severe valvular heart disease, atrial fibrillation, history of chronic or acute inflammatory disease in the preceding 3 months, malignancies, autoimmune diseases, and type $1 \mathrm{DM}$.

A written informed consent from all participants was taken.

All participants underwent the following:

I. Full history taking and clinical examination.

1. General examination: assessment of general condition and heart rate and blood pressure.

2. Cardiac examination for signs of heart failure, cardiac murmurs or cardiomegaly; pulse examination to detect peripheral vascular disease or significant arrhythmia.

3. Anthropometric measures: measurement of body weight in kilograms and height in meter, from which the body surface area (BSA) was calculated according to the formula of Dubois. ${ }^{17}$

$$
\begin{aligned}
&-\operatorname{BSA}\left(\mathrm{M}^{2}\right)= 0.007184 \times \text { HEIGHT }(\mathrm{CM})^{0.725} \times \\
& \text { WEIGHT }(\mathrm{KG})^{0.425} \\
& \text { 4. Echocardiography. }
\end{aligned}
$$

The M-mode measurements obtained included the following:

Two-dimensional guided M-mode measurements were obtained at the left lateral position from the parasternal long-axis view. Values were carefully obtained perpendicular to the LV long axis and measured at or immediately below the level of the mitral valve leaflet tips. In this regard, the electronic calipers were positioned on the interface between the myocardial wall and the cavity, and the interface between the wall and the pericardium, with special attention not to include the overlying trabeculations in the ventricular septum or posterior wall, which may overestimate thickness. Measurements were taken at end diastole (defined as the beginning of the QRS complex), but preferably using the widest LV cavity diameter, and at end systole using the narrowest LV cavity diameter.

- End-diastolic thickness of the ventricular septum (IVSD).

- End-diastolic thickness of the LV posterior wall (PWD).

- End-diastolic LV diameter (LVEDD).

- End-systolic LV diameter (LVEDS). 


\section{Assessment of LVM and LVMII}

Using the previous measured parameters, left ventricle mass (LVM) was calculated using the formula proposed by Devereux, the cube formula ${ }^{18}$ :

$$
\mathrm{LVM}=\left[1.04\left([\mathrm{LVEDD}+\mathrm{PWD}+\mathrm{IVSD}]^{3}-\mathrm{LVEDD}^{3}\right) \times 0.8\right]+0.6 .
$$

LVH was defined as left ventricular mass index (LVMI) $\geq 105 \mathrm{~g} / \mathrm{m}^{2}$ for men and $\geq 95 \mathrm{~g} / \mathrm{m}^{2}$ for women.

$$
\begin{aligned}
\operatorname{BSA}\left(\mathrm{M}^{2}\right)= & 0.007184 \times \operatorname{HEIGHT}(\mathrm{CM})^{0.725} \times \\
& \text { WEIGHT }(\mathrm{KG})^{0.425}
\end{aligned}
$$

\section{$\mathrm{LV} M \mathrm{MI}=\mathrm{LV} / \mathrm{M} / \mathrm{BSA}$}

1.04 is the specific gravity of the myocardium and 0.8 is a correction factor. LVMI $\left(\mathrm{g} / \mathrm{m}^{2}\right)$ was calculated by dividing the LVM $(\mathrm{g})$ value by BSA, which was calculated according to the formula of Dubois D and Dubois EF. ${ }^{17}$

\section{Assessment of LVEF}

LVEF was calculated according to the Simpson's formula:

$$
\mathrm{LVEF}=(\mathrm{LVEDV}-\mathrm{LVESV}) / \mathrm{LVEDV}
$$

where images were acquired from the apical fourchamber view at the left lateral position, and where LVEDV is left ventricular end-diastolic volume and LVESV is left ventricular end-systolic volume. ${ }^{19}$

\section{Laboratory investigations}

Blood samples were collected from all participants for routine laboratory investigations (complete blood count, fasting and 2-hour blood sugar, liver and kidney function tests, Prothrombin Time (PT), Activated Partial Thromboplastin Time (APTT) and lipid profile). Blood chemistry was done using a fully automated autoanalyzer Synchron CX9 ALX (Randox, California, USA), and hematology parameters were obtained using an automated Sysmex KX-21 (Sysmex Corporation, Kobe, Japan).

For lipid profile measurement, after an overnight fasting, total serum cholesterol, triglyceride, and highdensity lipoprotein cholesterol (HDL-c) were obtained using Synchron CX9 ALX (Randox), while low-density lipoprotein cholesterol (LDL-c) was estimated using Friedewald's formula.

IL-6, NT-proBNP and resistin were measured in all participants using ELISA (Chongqing Biospes, China). This kit was based on a sandwich ELISA technology.

\section{Statistical analysis}

SPSS V.25 computer program was used for statistical analysis. Numerical variables were expressed as mean and SD. Categorical variables were expressed as numbers and percentages. $F$ test was used to compare two variances by dividing them (it always tests whether population variance is equal).

Analysis of variance was performed to compare quantitative variables in more than two groups. $\mathrm{P}<0.05$ was statistically significant. Receiver operating characteristic (ROC) analysis was used to assess the diagnostic performance of the measured serum biomarkers for assessment of LVH and systolic dysfunction. Correlation analysis was done to assess the strength and direction of the relationship between quantitative variables.

The Pearson correlation coefficient was calculated to indicate the strength of association between nonnormally distributed numerical variables, where $r=1$ means a perfect positive correlation coefficient and $\mathrm{r}=-1$ means a perfect negative correlation coefficient.

\section{RESULTS}

Table 1 shows there was a statistically significant difference among the three studied groups with regard to diastolic blood pressure and duration of diabetes per year, where group 1a had higher level of diastolic blood pressure and duration of diabetes; however, no statistically significant difference was recorded with regard to systolic blood pressure, body mass index, height and waist circumference.

In terms of the laboratory investigations, there were statistically significant differences among the three studied groups with regard to fasting blood sugar (FBS), 2-hour post prandial blood sugar (2-hrs PPG) and LDL-c, which were higher in group 1a than in group $1 \mathrm{~b}$ by $18.81 \mathrm{mg} / \mathrm{dL}, 11.14 \mathrm{mg} / \mathrm{dL}$ and $10.14 \mathrm{mg} / \mathrm{dL}$, respectively, and HDL-c, which was lower in group 1a than in group $1 \mathrm{~b}$ by $8.76 \mathrm{mg} / \mathrm{dL}$. Meanwhile, no statistically significant differences were observed with regard to PT, total cholesterol, APTT and triglycerides (table 1).

The comparison between the DM subgroups and the control group in terms of echocardiographic parameters showed statistically significant differences between both DM subgroups with regard to Left Ventricular Posterior Wall Dimensions (LVPWD), interventricular septum (IVS), LVM, LVMI and Relative Wall Thickness (RWT), where all parameters were higher in group la by $11.2 \%$, $9.9 \%, 23.4 \%, 27.8 \%$ and $13.7 \%$, respectively, than in group $1 \mathrm{~b}$. There were statistically significant differences between the control group and group 1a with regard to left ventricle posterior wall thickness (LVPW), IVS, LVM, LVMI and RWT, where all parameters were higher in group 1a by $20.3 \%, 8 \%, 21.5 \%, 22.9 \%$ and $9.5 \%$, respectively, than in the control group. There was no statistically significant difference among all groups as regards LVEDD. There were no statistically significant differences between the control group and group $1 \mathrm{~b}$ as regards all echocardiographic parameters (table 2).

The comparison with regard to IL-6, resistin and NT-proBNP showed a statistically significant difference among all groups (P2). Also, there were statistically significant differences between group $1 \mathrm{a}$ and the other 2 groups, where the level of IL-6 in group 1a was $2.12,3.48$-fold than that of group $1 \mathrm{~b}$ and the control group, respectively; the level of resistin was higher in group $1 \mathrm{a}$ by 2.8 and $8.25 \mathrm{ng} / \mathrm{dL}$ than group $1 \mathrm{~b}$ and the control group, respectively; and NT-proBNP level was 2.23 and 7.47 folds higher in group 1a compared 
Table 1 Comparison among the three studied groups with regard to clinical and anthropometric parameters and laboratory investigations

\begin{tabular}{|c|c|c|c|c|c|c|c|}
\hline Parameters & Group & Mean & SD & Min & Max & $F$ & Significance \\
\hline \multirow[t]{3}{*}{ Systolic blood pressure } & Group 1a & 128.39 & 12.476 & 115 & 150 & 1.700 & 0.190 \\
\hline & Group 1b & 124.47 & 10.124 & 112 & 145 & & \\
\hline & Control group & 121.75 & 13.886 & 89 & 137 & & \\
\hline \multirow[t]{3}{*}{ Diastolic blood pressure } & Group 1a & 80.16 & 5.843 & 75 & 100 & 3.347 & $0.041^{*}$ \\
\hline & Group 1b & 75.26 & 6.118 & 78 & 95 & & \\
\hline & Control group & 77.75 & 7.860 & 60 & 85 & & \\
\hline \multirow[t]{3}{*}{ Waist circumference $(\mathrm{cm})$} & Group 1a & 78.52 & 11.243 & 42 & 101 & 1.548 & 0.220 \\
\hline & Group 1b & 113.44 & 142.915 & 61 & 702 & & \\
\hline & Control group & 77.08 & 18.151 & 8 & 98 & & \\
\hline \multirow[t]{3}{*}{ Height (cm) } & Group 1a & 164.26 & 7.598 & 149 & 183 & 0.859 & 0.428 \\
\hline & Group 1b & 167.37 & 11.329 & 148 & 188 & & \\
\hline & Control group & 166.45 & 7.141 & 155 & 180 & & \\
\hline \multirow[t]{3}{*}{ BMI } & Group 1a & 29.69 & 1.616 & 26 & 32 & 1.498 & 0.231 \\
\hline & Group 1b & 28.87 & 2.180 & 25 & 32 & & \\
\hline & Control group & 28.98 & 1.868 & 26 & 32 & & \\
\hline \multirow[t]{2}{*}{ DM (year) } & Group 1a & 5.47 & 1.807 & 3 & 9 & 91.045 & $0.021^{*}$ \\
\hline & Group 1b & 3.63 & 1.419 & 2 & 7 & & \\
\hline \multirow[t]{3}{*}{ Age (year) } & Group 1a & 47.71 & 3.752 & 40 & 57 & 1.656 & 0.199 \\
\hline & Group 1b & 44.89 & 3.365 & 39 & 52 & & \\
\hline & Control group & 45.05 & 10.242 & 37 & 55 & & \\
\hline \multirow[t]{3}{*}{ FBS (mg/dL) } & Group 1a & 141.23 & 28.888 & 95 & 210 & 31.589 & $0.006^{*}$ \\
\hline & Group 1b & 122.42 & 22.636 & 86 & 162 & & \\
\hline & Control group & 84.35 & 20.035 & 80 & 110 & & \\
\hline \multirow[t]{3}{*}{ 2hrs PPG (mg/dL) } & Group 1a & 196.03 & 35.489 & 148 & 300 & 65.316 & $0.001^{*}$ \\
\hline & Group 1b & 184.89 & 40.812 & 120 & 270 & & \\
\hline & Control group & 89.80 & 22.021 & 90 & 112 & & \\
\hline \multirow[t]{3}{*}{ PT (s) } & Group 1a & 11.61 & 0.803 & 11.5 & 13.5 & 1.961 & 0.149 \\
\hline & Group 1b & 11.11 & 1.049 & 11 & 13.7 & & \\
\hline & Control group & 11.40 & 0.821 & 10.5 & 13 & & \\
\hline \multirow[t]{3}{*}{ APTT (s) } & Group 1a & 38.06 & 0.509 & 38 & 42 & 6.254 & 0.06 \\
\hline & Group 1b & 39.68 & 0.478 & 37 & 4.3 & & \\
\hline & Control group & 39.30 & 0.470 & 36 & 37 & & \\
\hline \multirow[t]{3}{*}{ Total cholesterol (mg/dL) } & Group 1a & 191.10 & 54.111 & 113 & 306 & 2.925 & 0.061 \\
\hline & Group 1b & 167.53 & 33.414 & 110 & 226 & & \\
\hline & Control group & 163.50 & 35.447 & 85 & 178 & & \\
\hline \multirow[t]{3}{*}{ HDL-c (mg/dL) } & Group 1a & 49.03 & 9.635 & 43 & 60 & 14.689 & $0.001^{*}$ \\
\hline & Group 1b & 57.79 & 4.479 & 45 & 65 & & \\
\hline & Control group & 58.60 & 3.102 & 55 & 65 & & \\
\hline \multirow[t]{3}{*}{ LDL-c (mg/dL) } & Group 1a & 162.35 & 12.826 & 152 & 183 & 9.539 & $0.001^{*}$ \\
\hline & Group 1b & 152.21 & 10.438 & 140 & 170 & & \\
\hline & Control group & 69.85 & 7.659 & 55 & 106 & & \\
\hline \multirow[t]{3}{*}{ Triglyceride (mg/dL) } & Group 1a & 163.03 & 45.691 & 114 & 300 & 0.416 & 0.661 \\
\hline & Group 1b & 152.74 & 25.707 & 110 & 210 & & \\
\hline & Control group & 159.90 & 37.453 & 112 & 270 & & \\
\hline
\end{tabular}

*Significant $p$ value $(p<0.05)$

APTT, Activated Partial Thromboplastin Time; BMI, body mass index; DM, diabetes mellitus; FBS, fasting blood sugar; HDL-c, high-density lipoprotein cholesterol; 2hrs PPG, 2-hour post prandial blood sugar; LDL-c, low-density lipoprotein cholesterol; PT, Prothrombin Time. 
Table 2 Comparison between echocardiographic parameters in DM subgroups

\begin{tabular}{|c|c|c|c|c|c|c|}
\hline Parameters & $\begin{array}{l}\text { Group 1a }(n=46) \\
\text { Mean } \pm \text { SD }\end{array}$ & $\begin{array}{l}\text { Group 1b }(n=54) \\
\text { Mean } \pm S D\end{array}$ & $\begin{array}{l}\text { Control }(n=50) \\
\text { Mean } \pm \text { SD }\end{array}$ & P1 & P2 & P3 \\
\hline LVPWD & $\begin{array}{l}0.9774 \pm 0.16271 \\
(0.8-1.30)\end{array}$ & $\begin{array}{l}0.8789 \pm 0.09763 \\
(0.7-1.00)\end{array}$ & $\begin{array}{l}0.812 \pm 0.089 \\
(0.6-1.1)\end{array}$ & $0.021^{*}$ & $0.03^{*}$ & 0.1 \\
\hline IVS & $\begin{array}{l}0.9871 \pm 0.16682 \\
(0.7-1.30)\end{array}$ & $\begin{array}{l}0.9000 \pm 0.11055 \\
(0.7-1.00)\end{array}$ & $\begin{array}{l}0.914 \pm 0.101 \\
(0.7-0.9)\end{array}$ & $0.049^{*}$ & $0.02^{*}$ & 0.09 \\
\hline LVM & $\begin{array}{l}194.64 \pm 66.7776 \\
(18.060-333.72)\end{array}$ & $\begin{array}{l}157.062 \pm 25.8417 \\
(120.487-202.82)\end{array}$ & $\begin{array}{l}160.1 \pm 23.55 \\
(119.10-211.7)\end{array}$ & $0.023^{*}$ & $0.01^{*}$ & $0.02^{*}$ \\
\hline LVEDD & $\begin{array}{l}6.1677 \pm 8.1406 \\
(4.30-50.00)\end{array}$ & $\begin{array}{l}4.5579 \pm 0.18353 \\
(4.3-4.9)\end{array}$ & $\begin{array}{l}4.72 \pm 0.1791 \\
(4.5-5.5)\end{array}$ & 0.395 & 0.4 & 0.9 \\
\hline LVMI & $\begin{array}{l}104.752 \pm 29.0531 \\
(55.120-176.57)\end{array}$ & $\begin{array}{l}81.955 \pm 12.3183 \\
(56.490-97.082)\end{array}$ & $\begin{array}{l}85.21 \pm 14.00 \\
(54.91-98.19)\end{array}$ & $0.002^{*}$ & $0.012^{*}$ & 0.089 \\
\hline RWT & $\begin{array}{l}0.43803 \pm 0.06291 \\
(0.333-0.565)\end{array}$ & $\begin{array}{l}0.38584 \pm 0.04448 \\
(0.285-0.444)\end{array}$ & $\begin{array}{l}0.4 \pm 0.039 \\
(0.31-0.439)\end{array}$ & $0.047^{\star}$ & $0.035^{\star}$ & 0.4 \\
\hline EF & $\begin{array}{l}43 \pm 6.3 \\
(35-48)\end{array}$ & $\begin{array}{l}60 \pm 5.8 \\
(51-67)\end{array}$ & $\begin{array}{l}61 \pm 6.7 \\
(53-70)\end{array}$ & $0.001^{*}$ & $0.001^{*}$ & 0.437 \\
\hline
\end{tabular}

*means significant

DM, diabetes mellitus; EF, ejection fraction; IVS, interventricular septum; LVEDD, end-diastolic left ventricular diameter; LVM, left ventricle mass; LVMI, left ventricular mass index; LVPWD, Left Ventricular Posterior Wall Dimensions; P, Process; RWT, Relative Wall Thickness.

to both groups $1 \mathrm{~b}$ and the control group, respectively (table 3 and figure 1 ). There was no statistically significant difference among all groups with regard to gender (figure 2).

Table 4 shows there were statistically significant positive correlations between all biomarkers (NT-proBNP, IL-6, and resistin); a statistically significant inverse correlation between hemoglobin and all biomarkers except resistin; a statistically significant positive correlation between total cholesterol and both NT-proBNP and resistin; a statistically significant inverse correlation between HDL-c and both NT-proBNP and resistin; and a statistically significant positive correlation between triglycerides and NT-proBNP. Regarding echocardiographic parameters, there were statistically significant positive correlations between all biomarkers (NT-proBNP, IL-6, and resistin) and LVPW thickness, IVS thickness,
LVM, LVMI and RWT, while LVEDD had only statistically significant positive correlation with IL-6. There was no statistically significant difference among all groups as regards smoking, hypertension, obesity, gender, or drug use (table 5).

For discrimination between group $1 \mathrm{a}$ and group $1 \mathrm{~b}$ plus detection of LV systolic dysfunction in patients with T2DM, ROC analysis estimated a sensitivity of $100 \%$, $45.7 \%$, and $100 \%$, specificity of $61.1 \%, 81.5 \%$, and $92.6 \%$, positive predictive value of $68.7 \%, 67.8 \%$, and $92 \%$, negative predictive value of $100 \%, 63.8 \%$, and $100 \%$, and accuracy of $80.6 \%, 63.6 \%$, and $96.3 \%$, at the best cut-off value of $>8.8 \mathrm{pg} / \mathrm{mL},>22.14 \mathrm{ng} / \mathrm{mL}$ and $>444.13 \mathrm{pg} / \mathrm{dL}$ for IL-6, resistin and NT-proBNP, respectively, determined by calculating Youden index J. The area under the curve (AUC) was 0.852, 0.650, and 0.987, with a highly significant associated $\mathrm{p}<0.001$, significantly associated $\mathrm{p}<0.01$ and a highly significantly associated

Table 3 Comparison between the studied groups with regard to IL-6, resistin and NT-proBNP

\begin{tabular}{llcccc}
\hline Parameters & Group & Mean \pm SD & Range & P1 & P2 \\
\hline IL-6 $(\mathrm{ng} / \mathrm{mL})$ & Group 1a & $18.242 \pm 10.857$ & $8.9-66.6$ & $0.009^{*}$ & $0.001^{*}$ \\
& Group 1b & $8.563 \pm 2.370$ & $5.2-13.6$ & \\
& Control group & $5.240 \pm 1.697$ & $2.3-8.5$ & \\
Resistin $(\mathrm{ng} / \mathrm{mL})$ & Group 1a & $22.168 \pm 7.701$ & $12.70-42.21$ & $0.036^{*}$ & $0.001^{*}$ \\
& Group 1b & $19.368 \pm 4.222$ & $12.10-27.20$ & \\
NT-proBNP $(\mathrm{pg} / \mathrm{mL})$ & Control group & $13.935 \pm 3.080$ & $9.80-19.50$ & $0.0001^{*}$ & $0.001^{*}$ \\
& Group 1a & $652.39 \pm 163.700$ & $456-1026$ & $156-462$ & \\
\end{tabular}

*Significant $p$ value $(p<0.05)$.

IL-6, interleukin 6; NT-proBNP, NH2-terminal portion pro-brain natriuretic peptide; P, Process. 


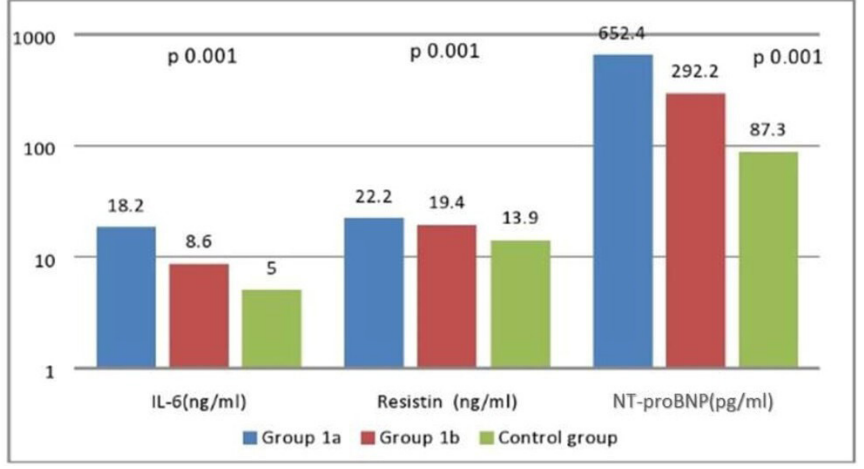

Figure 1 Comparison among the studied groups with regard to IL-6, resistin and NT-proBNP. There was a significant difference among the three groups, where all parameters were higher in group 1a. IL-6, interleukin-6; NTproBNP, NH2-terminal portion pro-brain natriuretic peptide.

$\mathrm{p}<0.001$ for IL-6, resistin and NT-proBNP, respectively (figure 3).

\section{DISCUSSION}

Studies about the role of proinflammatory cytokines in the pathogenesis of heart disease in T2DM are still not efficient. This study aimed to reveal the association between the circulating levels of three biomarkers (resistin, Nt-proBNP and IL-6) and LV systolic function in patients with T2DM and evaluate them as markers of LVD (left ventricular systolic dysfunction). One hundred and fifty participants participated in this study: 100 patients with T2DM and 50 healthy controls. Patients were divided after echocardiography into two subgroups: group 1a included 46 patients with T2DM and LVD (EF $<50 \%$ ), and group $1 \mathrm{~b}$ included 54 patients with T2DM and normal EF $(\mathrm{EF} \geq 50 \%)$.

This study found that there were statistically significant differences among the three studied groups with regard to diastolic blood pressure and duration of diabetes per year.

There were statistically significant differences among the three studied groups with regard to FBS, 2-hour PPBS

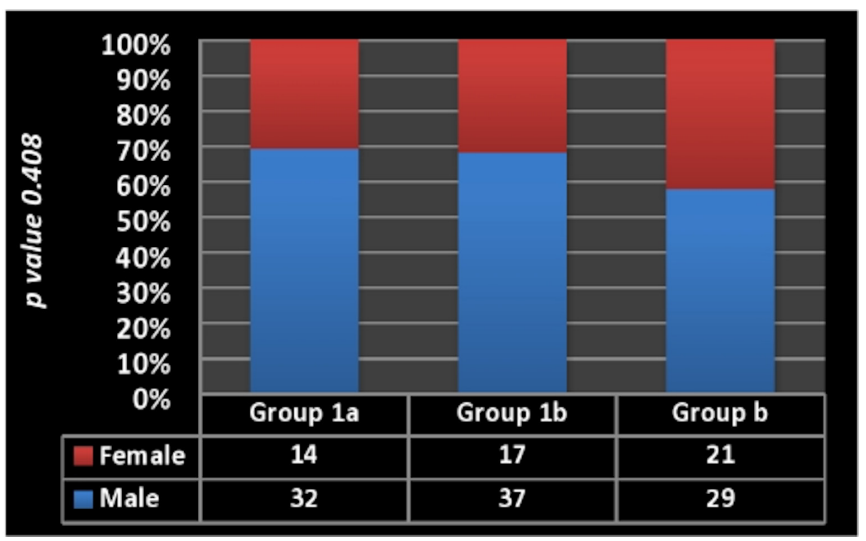

Figure 2 Gender distribution in all studied groups. There was no significant difference among the three groups as regards gender distribution.
Table 4 Correlation between NT-proBNP, IL-6, resistin, laboratory investigations and echocardiographic parameters in patients with diabetes

\begin{tabular}{|c|c|c|c|c|}
\hline Variables & & $\begin{array}{l}\text { Resistin } \\
\text { (ng/mL) }\end{array}$ & $\begin{array}{l}\text { IL-6 (ng/ } \\
\text { mL) }\end{array}$ & $\begin{array}{l}\text { NT- } \\
\text { proBNP } \\
\text { (pg/mL) }\end{array}$ \\
\hline \multirow{2}{*}{$\begin{array}{l}\text { NT-proBNP } \\
\text { (pg/mL) }\end{array}$} & $r$ & 0.970 & 0.685 & - \\
\hline & $\mathrm{P}$ value & $0.001^{*}$ & $0.001^{*}$ & - \\
\hline \multirow[t]{2}{*}{ IL-6 (ng/mL) } & $r$ & 0.685 & - & 0.667 \\
\hline & $\mathrm{P}$ value & $0.001^{*}$ & - & $0.001^{*}$ \\
\hline \multirow[t]{2}{*}{ Resistin (ng/mL) } & $r$ & - & 0.667 & 0.970 \\
\hline & $P$ value & - & $0.001^{*}$ & $0.001^{*}$ \\
\hline \multirow[t]{2}{*}{ White cell count } & $r$ & -0.027 & -0.011 & 0.012 \\
\hline & $\mathrm{P}$ value & 0.884 & 0.952 & 0.949 \\
\hline \multirow[t]{2}{*}{ Hemoglobin $(g / L)$} & $r$ & -0.343 & -0.331 & -0.415 \\
\hline & $\mathrm{P}$ value & 0.059 & $0.048^{*}$ & $0.020^{*}$ \\
\hline \multirow[t]{2}{*}{ Platelets } & $r$ & -0.201 & 0.026 & -0.193 \\
\hline & $\mathrm{P}$ value & 0.278 & 0.890 & 0.297 \\
\hline \multirow[t]{2}{*}{ FBS (mg/dL) } & $r$ & -0.168 & -0.204 & -0.152 \\
\hline & $P$ value & 0.367 & 0.270 & 0.416 \\
\hline \multirow{2}{*}{$\begin{array}{l}\text { 2hrs PPG (mg/ } \\
\mathrm{dL})\end{array}$} & $r$ & -0.192 & -0.234 & -0.137 \\
\hline & $P$ value & 0.300 & 0.206 & 0.463 \\
\hline \multirow[t]{2}{*}{ PT (s) } & $r$ & 0.054 & -0.042 & 0.096 \\
\hline & $\mathrm{P}$ value & 0.774 & 0.824 & 0.607 \\
\hline \multirow[t]{2}{*}{ APTT (s) } & $r$ & -0.017 & -0.023 & -0.045 \\
\hline & $\mathrm{P}$ value & 0.927 & 0.901 & 0.810 \\
\hline \multirow[t]{2}{*}{ Urea (mg/dL) } & $r$ & 0.111 & 0.287 & 0.045 \\
\hline & $P$ value & 0.552 & 0.117 & 0.809 \\
\hline \multirow{2}{*}{$\begin{array}{l}\text { Creatinine } \\
(\mathrm{mg} / \mathrm{dL})\end{array}$} & $r$ & 0.054 & -0.009 & 0.030 \\
\hline & $P$ value & 0.774 & 0.961 & 0.874 \\
\hline \multirow{2}{*}{$\begin{array}{l}\text { Total cholesterol } \\
\text { (mg/dL) }\end{array}$} & $r$ & 0.501 & -0.256 & 0.527 \\
\hline & $P$ value & $0.004^{*}$ & 0.165 & $0.002^{*}$ \\
\hline \multirow{2}{*}{$\begin{array}{l}\text { Triglyceride } \\
\text { (mg/dL) }\end{array}$} & $r$ & 0.356 & -0.150 & -0.308 \\
\hline & $P$ value & $0.049^{\star}$ & 0.422 & 0.092 \\
\hline \multirow[t]{2}{*}{ HDL-c (mg/dL) } & $r$ & -0.440 & -0.163 & -0.502 \\
\hline & $P$ value & $0.013^{\star}$ & 0.381 & $0.004^{*}$ \\
\hline \multirow[t]{2}{*}{ LDL-c (mg/dL) } & $r$ & -0.257 & -0.208 & -0.262 \\
\hline & $P$ value & 0.163 & 0.263 & 0.155 \\
\hline \multirow[t]{2}{*}{ LVEDD } & $r$ & 0.172 & 0.825 & 0.141 \\
\hline & $P$ value & 0.356 & $0.001^{*}$ & 0.451 \\
\hline \multirow[t]{2}{*}{ LVPW } & $r$ & 0.695 & 0.623 & 0.746 \\
\hline & $P$ value & $0.001^{*}$ & $0.001^{*}$ & $0.001^{*}$ \\
\hline \multirow[t]{2}{*}{ IVS } & $r$ & 0.719 & 0.617 & 0.752 \\
\hline & $P$ value & $0.001^{*}$ & $0.001^{*}$ & $0.001^{*}$ \\
\hline \multirow[t]{2}{*}{ LVM } & $r$ & 0.319 & 0.425 & 0.416 \\
\hline & $P$ value & $0.048^{*}$ & $0.017^{\star}$ & $0.020^{\star}$ \\
\hline \multirow[t]{2}{*}{ LVMI } & $r$ & 0.495 & 0.495 & 0.570 \\
\hline & $P$ value & $0.005^{\star}$ & $0.005^{\star}$ & $0.001^{*}$ \\
\hline
\end{tabular}


Table 4 Continued

\begin{tabular}{llccc}
\hline Variables & & $\begin{array}{l}\text { Resistin } \\
(\mathbf{n g} / \mathbf{m L})\end{array}$ & $\begin{array}{l}\text { IL-6 }(\mathbf{n g} / \\
\mathbf{m L})\end{array}$ & $\begin{array}{l}\text { NT- } \\
\text { proBNP } \\
(\mathbf{p g} / \mathbf{m L})\end{array}$ \\
\hline RWT & $r$ & 0.782 & 0.593 & 0.790 \\
& $P$ value & $0.001^{\star}$ & $0.001^{\star}$ & $0.001^{\star}$
\end{tabular}

*Significant $p$ value $(p<0.05)$.

APTT, Activated Partial Thromboplastin Time; FBS, fasting blood sugar; HDL-c, high-density lipoprotein cholesterol; 2hrs PPG, 2-hour post prandial blood sugar; IL-6, interleukin-6; IVS, interventricular septum; LDL-c, low-density lipoprotein cholesterol; LVEDD, end-diastolic left ventricular diameter; LVM, left ventricle mass; LVMI, left ventricular mass index; LVPW, left ventricle posterior wall; NT-proBNP, NH2-terminal portion pro-brain natriuretic peptide; PT, Prothrombin Time; RWT, Relative Wall Thickness.

(higher in group 1a), HDL-c (lower in group 1a), and LDL-c (higher in group 1a). Longer duration of DM with increased level of LDL and decreased HDL can explain the higher level of the proinflammatory markers in group 1a, and this is in agreement with Kim et $a l,{ }^{20}$ who stated that individuals with dyslipidemia have higher levels of inflammatory biochemical markers than those without dyslipidemia. Also, Lian $e t a l^{21}$ found that inflammation had a strong role in the development of dyslipidemia.

Consistent with our results, Magnusson et $a l^{22}$ found that patients with T2DM had higher NT-proBNP values than control subjects. NT-proBNP levels were independently related to diabetes after adjustment for age, sex, systolic and diastolic blood pressure, body mass index, heart rate, drug treatment, serum creatinine and cystatin C. The secretion of NT-proBNP was increased in patients with T2DM with no overt heart disease, suggesting that T2DM was associated with a higher prevalence of LVD and thus NT-proBNP might serve as a screening biomarker to select patients with T2DM who could benefit from an echocardiographic examination.

Richards $e t a l^{23}$ demonstrated that the combination of NT-proBNP and DM in patients with stable CHD provides independent prognostic information on allcause mortality. Dencker et $a l^{4}$ evaluated the relationship between natriuretic peptide levels and a wide range of echocardiography parameters in 32 patients with poorly regulated T2DM and unknown heart failure. They found a significant increase in the levels of both BNP and NT-proBNP in patients with abnormal diastolic function as compared with those with normal diastolic function.

Zhuravlyova and Sokolnikova ${ }^{25}$ reported a positive correlation of IL-6 and resistin with systolic and diastolic functions of the heart, increasing the functional and structural morbidity of the cardiac muscles.

Our study revealed statistically significant differences among the three studied groups with regard to biomarkers (NT-proBNP, IL-6, and resistin), with increased levels in group 1a. The inflammatory roles of these biomarkers and the longer duration of DM might

Table 5 Comparison among the three groups as regards risk factors and drug treatment, by $\chi^{2}$ test then one-way analysis of variance

\begin{tabular}{|c|c|c|c|c|c|}
\hline \multicolumn{2}{|c|}{ Risk factors and drug therapy } & \multirow{2}{*}{$\begin{array}{l}\text { Group 1a }(n=46) \\
32\end{array}$} & \multirow{2}{*}{$\begin{array}{l}\text { Group 1b }(n=54) \\
37\end{array}$} & \multirow{2}{*}{$\begin{array}{l}\text { Control group }(n=50) \\
29\end{array}$} & \multirow{2}{*}{$\begin{array}{l}\text { P value } \\
0.06\end{array}$} \\
\hline Male gender & $\mathrm{n}$ & & & & \\
\hline & $\%$ & 69.9 & 68.5 & 58.0 & \\
\hline \multirow[t]{2}{*}{ Smoking } & $\mathrm{n}$ & 31 & 30 & 22 & 0.4 \\
\hline & $\%$ & 67.4 & 55.6 & 44.0 & \\
\hline \multirow[t]{2}{*}{ Hypertension } & $\mathrm{n}$ & 27 & 20 & 0 & 0.4 \\
\hline & $\%$ & 57.8 & 37.9 & 0.0 & \\
\hline \multirow[t]{2}{*}{ Obesity $\left(\mathrm{BMI}>30 \mathrm{~kg} / \mathrm{m}^{2}\right)$} & $\mathrm{n}$ & 36 & 32 & 30 & 0.126 \\
\hline & $\%$ & 78.3 & 59.3 & 60 & \\
\hline \multirow[t]{2}{*}{ Aspirin } & $\mathrm{n}$ & 25 & 10 & 0 & 0.2 \\
\hline & $\%$ & 54.3 & 18.5 & 0.0 & \\
\hline \multirow[t]{2}{*}{ Beta blocker } & $\mathrm{n}$ & 40 & 8 & 3 & 0.6 \\
\hline & $\%$ & 87.0 & 14.8 & 6.0 & \\
\hline \multirow[t]{2}{*}{ ACEI } & $\mathrm{n}$ & 38 & 12 & 8 & 0.7 \\
\hline & $\%$ & 82.6 & 22.2 & 16.0 & \\
\hline \multirow[t]{2}{*}{ Statin } & $\mathrm{n}$ & 33 & 39 & 8 & 0.9 \\
\hline & $\%$ & 71.7 & 72.2 & 16.0 & \\
\hline \multirow[t]{2}{*}{ Diuretics } & $\mathrm{n}$ & 19 & 1 & 0 & 0.11 \\
\hline & $\%$ & 41.3 & 1.9 & 0.0 & \\
\hline
\end{tabular}

ACEI, ACE inhibitor; BMI, body mass index. 

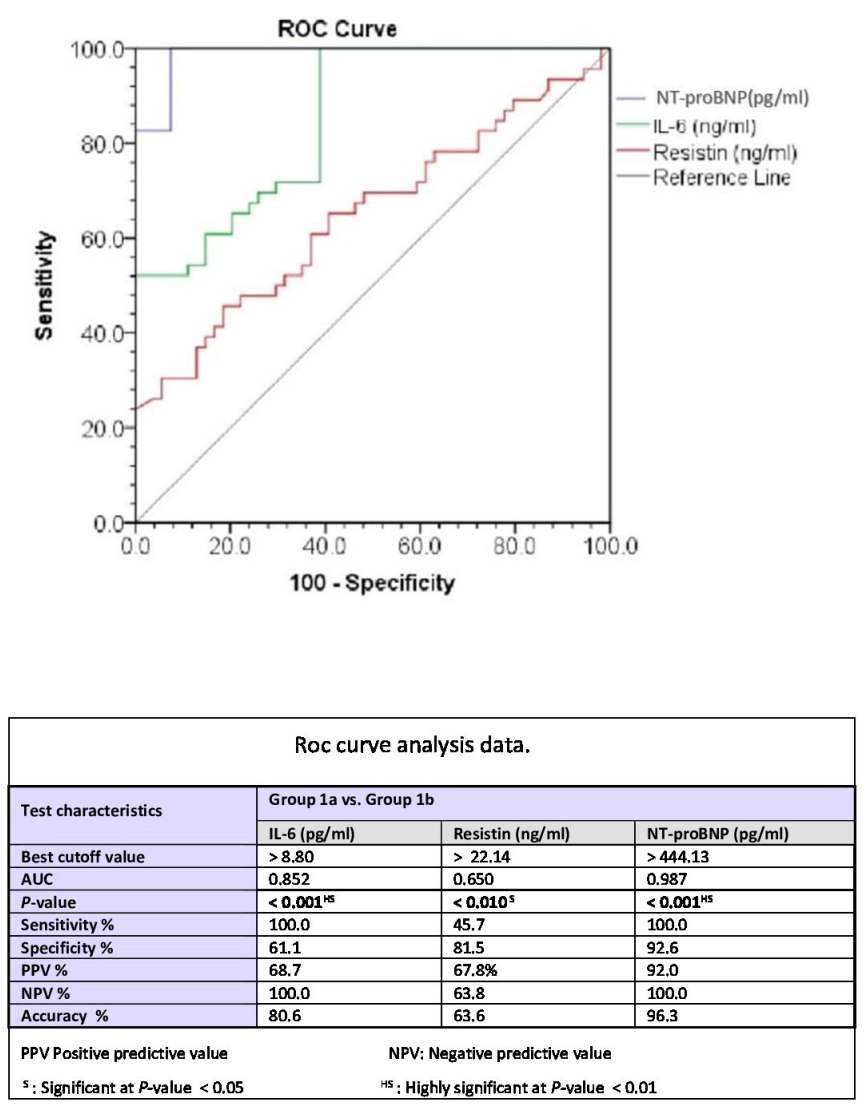

Figure 3 ROC curve analysis of IL-6, resistin, and NTproBNP with regard to detection of left ventricular systolic dysfunction. AUC, area under the curve; IL-6, interleukin-6; NT-proBNP, NH2-terminal portion pro-brain natriuretic peptide; $\mathrm{ROC}$, receiver operating characteristic.

explain their higher level in group 1a than in other groups and confirmed their role in the pathogenesis of $\mathrm{DM}$ as a chronic inflammatory disease.

Magnusson $e t a t^{22}$ reported higher NT-proBNP values in patients with T2DM than control subjects. In addition, Palazzuoli $e t a l^{8}$ discussed the correlation between the role of high plasma BNP in patients with systolic heart failure and the severity of diastolic abnormality.

Similarly, Shinohara et $a l^{11}$ suggested that inflammatory markers were independent predictors of cardiovascular events in older persons. IL-6, released in response to tissue injuries and infections, related to host defense through the stimulation of acute phase responses, hematopoiesis, and immune reactions. The high serum level of IL-6 had a predictive function in the pathogenesis of insulin resistance and cardiac complications in patients with diabetes. ${ }^{26-31}$

Also, Cheng $e t a l^{32}$ found that increased plasma concentrations of resistin were strongly associated with subsequent development of heart failure. The association of resistin with heart failure persisted after adjustment for established heart failure risk factors, obesity, markers of insulin resistance and inflammation, and concentrations of NT-proBNP, and after exclusion of prevalent and incident CHD. Erer $e t a l^{33}$ concluded that serum resistin concentration was elevated in patients with acute STEMI (sustained ST segment elevation of myocardial infarction), and this increase was more obvious in patients with T2DM than in those without diabetes.

Another finding in our study is the statistically significant positive correlation between all biomarkers (NT-proBNP, IL-6, and resistin) and myocardial indices (LVPW thickness, IVS thickness, LVM, LVMI and RWT), while LVEDD had only statistically significant positive correlation with IL-6. The relation between inflammation and cardiac remodeling and heart failure has been mentioned in many previous studies. ${ }^{4435}$

Valle et $a \hat{l}^{36}$ confirmed the diagnostic and prognostic role of BNP in systolic dysfunction evaluation.

Tuttolomondo et $a l^{12}$ explained the positive correlation between IL-6, resistin and vascular complications of DM. Zhuravlyova and Sokolnikova ${ }^{25}$ revealed a positive correlation between resistin, IL-6 and cardiac outcomes in T2DM, which played a role in the functional and structural morbidity of the cardiac muscles, reducing their systolic and diastolic functions.

The high level of resistin and IL- 6 was correlated with LVH and systolic dysfunction in Toczylowski et al. ${ }^{37}$

On ROC analysis of NT-proBNP, its cut-off level was $444.13 \mathrm{pg} / \mathrm{ml}$, with an AUC of $0.987(\mathrm{p}<0.001)$, sensitivity of $100 \%$ and specificity of $92.6 \%$, which mean that NT-proBNP is a novel, sensitive, specific predictor biomarker for T2DM.

Similarly, NT-proBNP ROC curve analysis by Castaño Rodríguez et $a l^{88}$ revealed a cut-off level of $490 \mathrm{pg} / \mathrm{mL}$ for detection of ischemia in patients with both T2DM and hypertension, which yielded an AUC of 0.864 , sensitivity of $84 \%$ and specificity of $75 \%$ in detecting LVH and systolic dysfunction; therefore, the level of NT-proBNP may be considered a promising biomarker for detection of Ischemic Heart Disease (IHD).

Kara $e t a l^{39}$ reported the superior role of NT-proBNP over BNP in early prediction of CHD. Baba et $a t^{40}$ explained the roles of nitro-BNP in the diagnosis and screening of CHD, atrial fibrillation and heart failure.

ROC analysis of IL-6 estimated an AUC of 0.852, sensitivity of $100 \%$, specificity of $61.1 \%$ and accuracy of $80.6 \%$ at the best cut-off value of $>8.8 \mathrm{pg} / \mathrm{mL}(\mathrm{p}<0.001)$. IL-6 is a good predictive, specific and sensitive marker.

Tuttolomondo et $a l^{12}$ reported that an IL-6 level $>4.8 \mathrm{pg} / \mathrm{mL}(\mathrm{p}<0.005)$ was significantly correlated with all outcomes (CHD events, stroke events and Congestive Heart Failure (CHF) events). Shinohara et $\mathrm{l}^{11}$ suggested the high predictive value of IL- 6 level $>2.5 \mathrm{pg} / \mathrm{mL}$ $(p<0.031)$ in screening for early cardiac complication in T2DM before a structural heart disease.

Similarly, Phosat $e t a t^{11}$ measured the levels of IL-6 and other inflammatory markers (tumor necrosis factor- $\alpha$, $\mathrm{C}$ reactive protein) and showed these were significantly associated with increased risk of diabetes.

The current study showed that ROC analysis of resistin recorded an AUC of 0.65 , sensitivity of $45.7 \%$, specificity of $81.5 \%$ and accuracy of $63.6 \%$, at the best cut-off 
value of $>22 \mathrm{ng} / \mathrm{mL}(\mathrm{p}<0.01)$. Therefore, resistin has good predictive value and high specificity, but with poor sensitivity.

\section{Limitations of this study}

We did not use a non-diabetic group with LV systolic dysfunction to compare their level of inflammatory markers with a diabetic group with systolic dysfunction, in order to confirm the effect of DM. We did not use new methods for assessment of subtle systolic dysfunction such as speckle tracking. Also we did not use the diastolic function assessment indices in the comparison between the studied groups.

\section{CONCLUSION}

The elevated levels of IL-6, NT-proBNP and resistin in T2DM support their possible role or inflammatory process in diabetogenesis. These markers may serve as non-invasive screening and new prospective prehypertrophic markers for LV remodeling and for detection of dysfunction, especially in asymptomatic patients with T2DM, and help to select patients with T2DM who could benefit from an echocardiographic examination. The specific mechanism whereby resistin and IL-6 promote CVD remains to be elucidated, and needs further study on a wide scale of population with long-term follow-up, as well as further genetic studies.

\section{Author affiliations}

${ }^{1}$ Department of Clinical Biochemistry and Molecular Diagnostics, National Liver Institute, Menoufia University, Menoufia, Egypt

${ }^{2}$ Department of Hepatology and Gastroenterology, National Liver Institute, Menoufia University, Menoufia, Egypt

${ }^{3}$ Department of Cardiology, Shibin Elkom Teaching Hospital, Menoufia, Egypt

${ }^{4}$ Department of Cardiology, Helwan University, Cairo, Egypt

${ }^{5}$ Department of Physiology, Faculty of Medicine for Girls, Al-Azhar University, Cairo, Egypt

${ }^{6}$ Department of Clinical Microbiology and Immunology and Molecular Microbiology in Liver and GIT, National Liver Institute, Menoufia University, Menoufia, Egypt ${ }^{7}$ Department of Clinical Pathology, National Liver Institute, Menoufia University, Menoufia, Egypt

${ }^{8}$ Department of Clinical Pharmacy, Faculty of Pharmacy, Menoufia University, Menoufia, Egypt

${ }^{9}$ Department of Epidemiology and Preventive Medicine, National Liver Institute, Menoufia University, Menoufia, Egypt

${ }^{10}$ Department of Internal Medicine, Faculty of Medicine, Menoufia University,

Menoufia, Egypt

${ }^{11}$ Department of Internal Medicine, Helwan University, Cairo, Egypt

Acknowledgements The authors have the pleasure of dealing with the journal and would like to thank and hope that this research work would be under their kind care and observation.

Contributors All authors made substantial contributions to conception and design, acquisition of data, or analysis and interpretation of data; took part in drafting the article or revising it critically for important intellectual content; gave final approval of the version to be published; and agree to be accountable for all aspects of the work.

Funding The authors have not declared a specific grant for this research from any funding agency in the public, commercial or not-for-profit sectors.

Competing interests None declared.

Patient consent for publication Not required.
Ethics approval The study was reviewed and approved by the ethical committee at the National Liver Institute (IRB number 00199), Menoufia University. The study was conducted in accordance with Good Clinical Practice guidelines and the Declaration of Helsinki after local ethical committee approval.

Provenance and peer review Not commissioned; externally peer reviewed.

Data availability statement № data are available.

Open access This is an open access article distributed in accordance with the Creative Commons Attribution Non Commercial (CC BY-NC 4.0) license, which permits others to distribute, remix, adapt, build upon this work non-commercially, and license their derivative works on different terms, provided the original work is properly cited, appropriate credit is given, any changes made indicated, and the use is non-commercial. See: http://creativecommons.org/licenses/by-nc/4.0/.

ORCID iD

Mohamed Abdel-Samiee http://orcid.org/0000-0002-8970-0286

\section{REFERENCES}

1 American heart association cardiovascular diseases and diabetes (AHAC) 2015

2 Holland DJ, Marwick TH, Haluska BA, et al. Subclinical LV dysfunction and 10-year outcomes in type 2 diabetes mellitus. Heart 2015;101:1061-6.

3 Kurioka S, Ose H, Fukuma K, et al. Severity of diabetic retinopathy is associated with left ventricular diastolic dysfunction in patients with type 2 diabetes. Diabetes Res Clin Pract 2013;99:287-91.

4 Fathelbaba M, Fahmyb EM, Elshormilisyb AA, et al. A putative role for oxidative stress in athophysiology of diabetic cardiomyopathy. Egypt J Obes Diabetes Endocrinol 2017;3:95-9.

5 Ofstad AP. Myocardial dysfunction and cardiovascular disease in type 2 diabetes. Scand J Clin Lab Invest 2016;76:271-81.

6 Madamanchi C, Alhosaini H, Sumida A, et al. Obesity and natriuretic peptides, BNP and NT-proBNP: mechanisms and diagnostic implications for heart failure. Int J Cardiol 2014;176:611-7.

7 Geng Z, Huang L, Song M, et al. N-Terminal pro-brain natriuretic peptide and cardiovascular or all-cause mortality in the general population: a meta-analysis. Sci Rep 2017;7:41504.

8 Palazzuoli M, Gallotta I, Quatrini R, et al. Natriuretic peptides (BNP and NT-pro BNP): measurement and relevance in heart failure. Vasc Health Risk Manag 2010;6:411-8.

9 Patil VC, Patil HV, Shah KB, et al. Diastolic dysfunction in asymptomatic type 2 diabetes mellitus with normal systolic function. $J$ Cardiovasc Dis Res 2011;2:213-22.

10 Wainstein MV, Mossmann M, Araujo GN, et al. Elevated serum interleukin-6 is predictive of coronary artery disease in intermediate risk overweight patients referred for coronary angiography. Diabetol Metab Syndr 2017;9:67.

11 Shinohara Tet al. Interleukin-6 as an independent predictor of future cardiovascular events in patients with type-2 diabetes without structural heart disease. J Clin Exp Cardiolog 2012;03:9.

12 Tuttolomondo A, La Placa S, Di Raimondo D, et al. Adiponectin, resistin and IL-6 plasma levels in subjects with diabetic foot and possible correlations with clinical variables and cardiovascular comorbidity. Cardiovasc Diabetol 2010;9:50.

13 Abate N, Sallam HS, Rizzo M, et al. Resistin: an inflammatory cytokine. Role in cardiovascular diseases, diabetes and the metabolic syndrome. Curr Pharm Des 2014;20:4961-9.

14 Menzaghi C, Marucci A, Antonucci A, et al. Suggestive evidence of a multi-cytokine resistin pathway in humans and its role on cardiovascular events in high-risk individuals. Sci Rep 2017;7:44337.

15 Marques-Vidal P, Bastardot F, von Känel R, et al. Association between circulating cytokine levels, diabetes and insulin resistance in a population-based sample (CoLaus study). Clin Endocrinol 2013;78:232-41.

16 American diabetes association: standard of medical care in diabetes 2019. Diabetes Care 2019;42:S4-6.

17 Dubois D, Dubois EF. A formula to estimate the approximate surface area if height and weight be known. Arch Int Med 1916;17:863-71.

18 Devereux RB. Detection of left ventricular hypertrophy by M-mode echocardiography. anatomic validation, standardization, and comparison to other methods. Hypertension 1987;9:9-26.

19 Hendel RC, Patel MR, Kramer CM, et al. ACCF/ACR/SCCT/SCMR/ ASNC/NASCI/SCAI/SIR 2006 appropriateness criteria for cardiac computed tomography and cardiac magnetic resonance imaging: a report of the American College of cardiology Foundation quality strategic directions Committee appropriateness criteria Working group, American College of radiology, society of cardiovascular computed tomography, Society for cardiovascular magnetic 
resonance, American Society of nuclear cardiology, North American Society for cardiac imaging, Society for cardiovascular angiography and interventions, and society of interventional radiology. J Am Coll Cardiol 2006;48:1475-97.

$20 \mathrm{Kim} \mathrm{M-H,} \mathrm{Kim} \mathrm{H-N,} \mathrm{Choi} \mathrm{W-S.} \mathrm{The} \mathrm{association} \mathrm{between} \mathrm{subclinical}$ inflammation and abnormal glucose and lipid metabolisms in normal-weight Korean individuals. Nutr Metab Cardiovasc Dis 2018;28:1106-13.

21 Lian Y, Xie L, Liu Y, et al. Metabolic-related markers and inflammatory factors as predictors of dyslipidemia among urban Han Chinese adults. Lipids Health Dis 2019;18:167.

22 Magnusson M, Melander O, Israelsson B, et al. Elevated plasma levels of NT-proBNP in patients with type 2 diabetes without overt cardiovascular disease. Diabetes Care 2004;27:1929-35.

23 Richards M, Nicholls MG, Espiner EA, et al. Comparison of Btype natriuretic peptides for assessment of cardiac function and prognosis in stable ischemic heart disease. J Am Coll Cardiol 2006;47:52-60.

24 Dencker M, Stagmo M, Dorkhan M. Relationship between natriuretic peptides and echocardiography parameters in patients with poorly regulated type 2 diabetes. Vasc Health Risk Manag 2010;6:373-82.

25 Zhuravlyova L, Sokolnikova N. 0149: the relationship between resistin, interleukin-6 level and diastolic dysfunction in patients with type 2 diabetes mellitus. Arch Cardiovasc Dis Suppl 2015;7:33.

26 El-Shafie M, Allam H, El-Shall L, et al. Nuclear factor-kappa B expression and Acyl-Ghrelin in Egyptian patients with non-alcoholic fatty liver. World J Res Rev;4:1-7.

27 Hendy OM, Rabie H, El Fouly A, et al. The circulating microRNAs (-122, -34a and -99a) as predictive biomarkers for non-alcoholic fatty liver diseases. Diabetes Metab Syndr Obes 2019;12:2715-23.

28 Abdelwahab SF, Zakaria Z, Sobhy M, et al. Differential distribution of IL28B.rs12979860 single-nucleotide polymorphism among Egyptian healthcare workers with and without a hepatitis $C$ virus-specific cellular immune response. Arch Virol 2015;160:1741-50.

29 Bakeer MS, Youssef MI, Elshazly HM, et al. On-treatment improvement of an emerging psychosomatic depressive disorder among Salmonella carriers: a multicenter experience from Egypt. Infect Drug Resist 2019;12:2573-82.
30 Hamdy S, Osman AM, Zakaria ZA, et al. Association of Toll-like receptor 3 and Toll-like receptor 9 single-nucleotide polymorphisms with hepatitis $C$ virus persistence among Egyptians. Arch Virol 2018:163:2433-42.

31 Abdelwahab SF, Zakaria Z, Allam WR, et al. Interleukin 28B. rs12979860 genotype does not affect hepatitis C viral load in Egyptians with genotype 4 chronic infection. Arch Virol 2015;160:2833-7.

32 Cheng JM, Akkerhuis KM, Battes LC, et al. Biomarkers of heart failure with normal ejection fraction: a systematic review. Eur $J$ Heart Fail 2013;15:1350-62.

33 Erer HB, Sayar N, Guvenc TS, et al. Prognostic value of serum resistin levels in patients with acute myocardial infarction. Kardiol Pol 2014;72:181-6.

34 Van Linthout S, Tschöpe C. Inflammation - Cause or Consequence of Heart Failure or Both? Curr Heart Fail Rep 2017; 14:251-65.

35 Riehle C, Bauersachs J. Key inflammatory mechanisms underlying heart failure. Herz 2019;44:96-106.

36 Valle R, Bagolin E, Canali C, et al. The BNP assay does not identify mild left ventricular diastolic dysfunction in asymptomatic diabetic patients. Eur J Echocardiogr 2006;7:40-4.

37 Toczylowski K, Hirnle T, Harasiuk D, et al. Plasma concentration and expression of adipokines in epicardial and subcutaneous adipose tissue are associated with impaired left ventricular filling pattern. $J$ Transl Med 2019;17:310.

38 Castaño Rodríguez S, Coma-Canella I, López Salazar B, et al. Echocardiographic findings and NT-proBNP level in type-2 diabetic patients with and without ischemic heart disease. Rev Esp Cardiol 2009;62:1184-8.

39 Kara K, Lehmann N, Neumann T, et al. NT-proBNP is superior to BNP for predicting first cardiovascular events in the general population: the Heinz Nixdorf recall study. Int $J$ Cardiol 2015;183:155-61.

40 Baba M, Yoshida K, leda M. Clinical applications of natriuretic peptides in heart failure and atrial fibrillation. Int $\mathrm{J} \mathrm{Mol} \mathrm{Sci}$ 2019;20:2824.

41 Phosat C, Panprathip P, Chumpathat N, et al. Elevated C-reactive protein, interleukin 6 , tumor necrosis factor alpha and glycemic load associated with type 2 diabetes mellitus in rural Thais: a crosssectional study. BMC Endocr Disord 2017;17:44. 